\title{
O modelo de cuidado centrado no paciente sob a perspectiva do paciente idoso
}

The patient-centered care model from the perspective of the elderly patient

El modelo de cuidado centrado en el paciente desde la perspectiva del paciente mayor

Denise Gonçalves de Araújo Mello Paranhos ${ }^{1}$ Aline Albuquerque Sant'Anna Oliveira ${ }^{2}$

RESUMO. Objetivo: Debate-se cada vez mais na esfera acadêmica a necessidade de mudança do padrão tradicional de cuidados, assimétrico e paternalista, por um modelo centrado no paciente, em que este, reconhecido como ator central dos cuidados, é estimulado pelos profissionais da saúde e familiares a participar ativamente de sua terapêutica. Esse trabalho centra-se no paciente idoso por enfrentar problemas de saúde mais frequentes e, em razão de sua vulnerabilidade acrescida, sujeitar-se a violações de direitos no cenário dos cuidados. O objetivo é discutir a adoção de um modelo de cuidado centrado no paciente idoso que atenda às suas especificidades e seja capaz de habilitá-lo a se autodeterminar. Metodologia: Essa investigação se fundamentou no levantamento, sistematização e análise de artigos científicos sobre modelos de cuidados centrados no paciente. Inicialmente, apresentou-se uma perspectiva geral sobre a doença para, a seguir, discutir-se a estipulação de uma base conceitual para cuidados centrados no paciente, a partir de discursos diversos contidos na literatura científica. Por fim, a partir das peculiaridades do paciente idoso, buscou-se compreender sua posição frente à doença para se pensar na instituição de um modelo de cuidados para ele moldado e que o reconheça como ator principal do tratamento. Conclusão: $O$ paciente idoso pode ser um protagonista ativo de sua terapêutica, desde que o modelo de cuidado oferecido o reconheça como ser vulnerável e central do tratamento, possa capacitá-lo a participar dos cuidados, compartilhar decisões e se autodeterminar.

Palavras-chave: Assistência Centrada no Paciente. Idoso. Bioética. Direitos Humanos.

ABSTRACT. Objective: It has been highly discussed in the academic sphere the need to change the traditional standard of care, asymmetric and paternalistic, for a patient-centered model, in which the patient, recognized as the central actor of care, is stimulated by health professionals and family members to actively participate in their therapy. This paper

\footnotetext{
${ }^{1}$ Doutora em Bioética pela Universidade de Brasília, Cátedra Unesco. Especialista em Direito Tributário pela Pontifícia Universidade Católica de Goiás. Graduada em Direito pela Universidade Federal de Goiás. Analista Judiciário da Justiça Federal, Seção Judiciária de Goiás.

2 Pós-Doutorado em Direitos Humanos e Pesquisadora Visitante do Centro de Direitos Humanos da Universidade de Essex, Inglaterra. Pós-Doutorado em Direito Humano à Saúde e Pesquisadora Visitante no Instituto de Direitos Humanos da Universidade de Emory, Estados Unidos. Professora Credenciada da PósGraduação em Bioética da Universidade de Brasília e do Curso de Especialização em Bioética da Cátedra UNESCO de Bioética. Membro do CBEC. Professora de Direitos Humanos do UniCEUB. Professora do Curso de Especialização em Direito Médico da UERJ, do Curso de Especialização em Direito Sanitário da FIOCRUZ. Professora de Direitos Humanos da Pós-Graduação do UniCEUB. Pesquisadora Associada do Núcleo de Diplomacia e Saúde da FIOCRUZ. Advogada da União. Brasília/DF.
} 
focuses on the elderly patients because they face more frequent health problems and, due to their increased vulnerability, are subject to violations of rights in the care setting. The objective is to discuss the adoption of a patient-centered care model for elderly patients that meets their specificities and enables them to be self-determined. Methodology: This research was based on the survey, systematization and analysis of scientific studies on models of patient-centered care. Initially, a general perspective on the disease was presented, and a stipulation of a conceptual basis for patient-centered care was discussed, based on several discourses contained in the scientific literature. Finally, based on the peculiarities of the elderly patients, we sought to understand their position in relation to the disease, in order to think about the institution of a model of care shaped for them, in which they are recognized as the principal actor of the treatment. Conclusion: It was concluded that elderly patients can be active protagonists of their therapy, if the model of care offered recognizes them as vulnerable, as the central actor of the treatment, and enables them to share decisions and to be self-determined.

Keywords: Patient-Centered Care. Aged. Bioethics. Human Rights.

RESUMEN. Objetivo: Se discute cada vez más en la esfera académica la necesidad de cambiar el patrón tradicional de cuidados, asimétrico y paternalista, por un modelo centrado en el paciente, en que éste, reconocido como actor central de los cuidados, es estimulado por los profesionales de la salud y familiares a participar activamente en su tratamiento. Este trabajo se centra en el paciente mayor por enfrentarse a problemas de salud más frecuentes y, debido a su mayor vulnerabilidad, someterse a violaciones de derechos en el escenario de los cuidados. El objetivo es discutir la adopción de un modelo de cuidado centrado en el paciente mayor que atienda a sus especificidades y sea capaz de habilitarlo a autodeterminarse. Metodología: Esta investigación se fundamentó en el levantamiento, sistematización y análisis de artículos científicos sobre modelos de cuidados centrados en el paciente. Inicialmente, se presentó una perspectiva general sobre la enfermedad para, a continuación, discutir la estipulación de una base conceptual para cuidados centrados en el paciente, a partir de discursos diversos contenidos en la literatura científica. Por último, a partir de las peculiaridades del paciente anciano, se buscó comprender su posición frente a la enfermedad para pensar en la institución de un modelo de cuidados para él moldeado y que lo reconozca como actor principal del tratamiento. Conclusión: El paciente anciano puede ser un protagonista activo de su terapéutica, siempre que el modelo de cuidado ofrecido lo reconozca como ser vulnerable y central del tratamiento, pueda capacitarlo a participar del cuidado, compartir decisiones y autodeterminarse.

Palabras-Illave: Atención Dirigida al Paciente. Anciano. Bioética. Derechos humanos.

\section{Introdução}

O paciente idoso, por suas especificidades, deve gozar de cuidados em saúde que levem em conta suas perdas físicas, cognitivas, sua maior lentidão em entender as terapêuticas que lhes são propostas e as dificuldades em decidir sobre os cursos de ação face aos tratamentos disponíveis. Muito se discute sobre a necessidade de mudança do modelo tradicional de cuidados, assimétrico e paternalista, por um modelo centrado no 
paciente, no qual se permitiria maior participação do doente na terapêutica e 0 compartilhamento de decisões. Entretanto, se não é fácil atingir um ideal de cuidados que envolva de forma simétrica profissionais da saúde e pacientes em geral, tal perspectiva se agrava em relação ao paciente idoso, que faz parte de um grupo de pessoas com vulnerabilidade acrescida e usualmente tido como incapaz de se autodeterminar.

A partir das últimas décadas, um apelo universal tem sido difundido pelas instituições de saúde em torno do mundo acerca da necessidade de adoção de um modelo de cuidados centrado no paciente (1), em razão do entendimento de que a centralidade dos cuidados beneficia os aspectos sociais, psicológicos, culturais e éticos das relações em saúde (2). Embora a adoção de um modelo de cuidados que centre a atenção nos pacientes tenha se tornado algo desejável, não existe um conceito universal e padrões bem definidos sobre como aplicá-lo na prática, na medida em que o processo de adoecimento é algo complexo e é experimentado por cada ser humano de forma diversa.

Os cuidados em saúde são geralmente motivados por certo grau de esperança, ou seja, o paciente tem esperança de cura, que sua dor seja aliviada e que poderá voltar para casa. No mesmo sentido, os profissionais de saúde esperam contribuir para a prevenção de doenças, apresentar um diagnóstico acertado e oferecer um tratamento eficaz. Entretanto, os profissionais de saúde têm dificuldades em lidar com a esperança do paciente e, muitas vezes, para não frustrar suas expectativas, omitem más notícias ou prestam informações incompletas ou inadequadas, o que viola o direito do paciente de ser informado e de participar das decisões sobre os cuidados (3).

No modelo de cuidado em que a preocupação central é com o paciente, este necessita identificar o que, no seu íntimo, realmente busca num encontro médico, como, por exemplo, se objetiva orientações, prevenir doenças, o alívio da dor, o conhecimento dos riscos da doença e seus prognósticos, possibilidade de cura ou de mais tempo de vida. As relações em saúde são intrincadas, razão pela qual o profissional deve ultrapassar as barreiras do tecnicismo e da ciência para atingir a linguagem do paciente, compreender suas necessidades, desejos e expectativas, estabelecer uma relação de confiança, informar de forma adequada, ouvir e acolher o paciente como ser único e central da terapêutica, e a quem devem ser conferidos os melhores cuidados em saúde (3).

Atualmente, estimula-se cada vez mais a diminuição das assimetrias na relação de cuidados em saúde, mediante o envolvimento do paciente no tratamento e o 
compartilhamento das decisões entre profissionais da saúde, pacientes e familiares. Acredita-se que decisões tomadas em conjunto trazem à tona o real desejo do paciente. Entretanto, uma das grandes dificuldades para a diminuição das assimetrias e maior compartilhamento de cuidados reside nos dissensos existentes entre o que o paciente quer e o que o profissional de saúde julga mais adequado (4).

O presente trabalho visa analisar o modelo de cuidado centrado no paciente sob a perspectiva do paciente idoso. Sob o prisma metodológico, esta investigação se fundamentou no levantamento, sistematização e análise de artigos científicos sobre modelos de cuidados centrados no paciente. Assim, num primeiro momento, apresentouse uma perspectiva geral sobre a doença para, posteriormente, discutir-se a estipulação de uma base conceitual para cuidados centrados no paciente, a partir de discursos diversos contidos na literatura científica. Por fim, a partir das peculiaridades do paciente idoso, buscou-se compreender sua posição frente à doença, levando-se em conta sua vulnerabilidade acrescida, para se pensar na instituição de um modelo de cuidados para ele moldado e que o considere como ator principal do tratamento. O estudo tem como foco o paciente idoso por integrar grupo vulnerável mais propenso a enfrentar problemas de saúde e abusos nas relações de cuidados.

Esclarece-se que o emprego do termo modelo valeu-se da nomenclatura utilizada pela Organização Mundial de Saúde (5) que, ao tratar dos direitos dos pacientes, enumerou diferentes modelos de cuidado possíveis entre pacientes e profissionais da saúde. Passa-se, inicialmente, à discussão de alguns aspectos da doença, com destaque à necessidade de, diante dela, buscar-se um padrão de cuidados centrado nas necessidades e desejos de cada paciente.

\section{O enfrentamento da doença pelo paciente}

Para se compreender a posição do paciente face à doença é necessário, antes, não só defini-la, mas contextualizá-la. Com esse objetivo, adotou-se Berlinguer, bioeticista italiano, que trabalhou aspectos importantes sobre a doença, capazes de incitar reflexões sobre o que ela representa para o doente; os sentimentos que nos suscita; bem como o que expressa em termos de sofrimento, de diversidade e até de estímulo. A partir do estudo de Berlinguer sobre a doença, os fatores que a cercam e os modos de se enfrentála, serão colhidos elementos para discussão sobre as relações entre os profissionais da 
saúde e seus pacientes, bem como para reflexão acerca dos modelos de cuidado modernamente propostos e que têm como objetivo colocar o paciente em plano central, de modo a torná-lo um participante ativo de sua terapêutica.

Berlinguer (6) relata que uma atribuição que sempre foi conferida aos profissionais da saúde é a de combater a doença e que esta é cercada de muitas definições e poucas certezas, sendo encarada de forma diversa por cada doente, por seus familiares e amigos, e pelos próprios profissionais. $O$ autor reconhece que não há uma definição suficientemente adequada para doença, motivo pelo qual assevera que devemos "partir dos sofrimentos, das diferenças e anomalias, dos perigos, dos sinais e dos estímulos que cada processo patológico desencadeia e influi na vida dos indivíduos e nas relações sociais" (6). A palavra 'doença' exprime um sentimento de perturbação, mal-estar, dor, dessa forma, a pessoa doente seria aquela que precisa de atenção individualizada e de tratamento, bem como de estímulo, condutas de solidariedade, tolerância e integração. Ciente das dificuldades de se estabelecer um conceito preciso sobre a doença, Berlinguer (6) propõe que nos coloquemos no lugar do paciente para que reflitamos sobre como a doença é vivida e enfrentada; quais as consequências para o doente e que sentimentos e comportamentos suscitam nos que o cercam; como se dá o sofrimento, se encara a diversidade, se enfrenta o perigo, se identificam os sinais e se possibilita o estímulo. Segundo o autor, o desrespeito ao paciente gera mais sofrimento do que a própria doença e destaca que o importante não é agir contra o doente, mas sim contra a doença, e que se deve "tratar da doença em cada doente" (6).

$\mathrm{Na}$ evolução das ciências médicas é possível constatar que apesar das conquistas obtidas com o desenvolvimento técnico-científico, surgiram também exageros e deformações capazes de levar as tecnologias a perderem de vista o paciente em sua complexidade. De fato, o processo de cuidados não envolve apenas o uso de aparelhos e medicamentos, mas deve levar em conta estilos de vida, comportamentos, valores e a participação do paciente em sua terapêutica. A participação do paciente é importante porque o restabelecimento envolve a conjugação de três fatores: "a força medicamentosa da natureza (...), a ajuda da ciência (...) e a determinação consciente do doente" (6).

Reconhece-se, assim, o poder excessivo conferido à classe médica e a necessidade de correção das distorções das relações de cuidados, de afastamento do papel de agente regulador e central desempenhado pelos profissionais da saúde em prol de uma relação 
em que os pacientes são tratados de igual maneira, diferindo apenas em relação à diversidade dos quadros clínicos. Numa relação adequada de cuidados, o profissional da saúde, além de prescrever, deve saber escutar, informar e inserir o doente de forma ativa na terapêutica, de modo que a relação não se volte só para o tratamento, mas também para a prevenção, reabilitação e melhoria da capacidade das pessoas (7). Deve haver uma relação de compartilhamento dos cuidados, pois "o paciente, que no processo saúdedoença deve ser considerado sujeito ético, pelo menos tanto quanto o médico corre o risco de ser relegado à posição passiva e subalterna" (7).

Embora Berlinguer encarasse a doença a partir de um ponto de vista coletivo e suas ações estivessem voltadas às políticas públicas de erradicação, previsão e prevenção das doenças como forma de melhor saúde para todos, o bioeticista não perdeu de vista questões individuais que compõem o adoecer, o sofrimento único por ela gerado, 0 processo pessoal de se enfrentá-la e a necessidade de correção das distorções das relações de cuidado. Com efeito, fatores diversos devem ser levados em conta num processo de adoecimento. Quando o foco do profissional da saúde é meramente na doença, corre-se o risco de obter diagnósticos equivocados e de se utilizar tratamentos ineficientes $(1,8)$. O paciente não pode ser reduzido à sua doença, pois a dignidade humana não está atrelada às condições de vida ou ao julgamento de terceiros (9). Assim, acredita-se que por meio de cuidados centrados no paciente é possível minorar os efeitos físicos da doença, compensar perdas cognitivas, propiciar um conhecimento de si e uma revisão de valores, bem como contornar os efeitos sociais que envolvem o processo de adoecimento. Com base em tal assertiva parte-se, assim, à discussão de modelos de cuidados centrados no paciente, presentes na literatura científica.

\section{Modelos de cuidados centrados no paciente}

Embora se reconheça a importância da adoção de um modelo de cuidado centrado no paciente, é consenso entre os pesquisadores que muito ainda se tem a fazer, tanto em termos de definições e conceitos, quanto em questão de estipulação de padrões de cuidados de fato centrados no paciente e que possam ser porventura amplamente aceitos $(10,11,12,13)$. Apesar da definição ainda obscura, existe um considerável consenso com relação aos atributos e fatores organizacionais necessários para se estabelecer cuidados centrados no paciente (14). No entanto, a diversidade dos discursos acerca da 
centralidade dos cuidados é inevitável, mas, ao mesmo tempo, necessária e valiosa, pois ajuda os profissionais da saúde e pacientes a se posicionarem e a construírem particularmente suas relações (13).

Apesar de vários elementos em comum nortearem a ideia de cuidados centrados no paciente, o modelo é relativamente novo e ainda contempla dissensos quanto à sua aplicação. Entre as décadas de 1960 e 1970, ocorreram várias mudanças nas práticas médicas, dentre elas a forma de relacionamento entre profissionais da saúde e pacientes. A partir desse período, o paternalismo começou a ser questionado por uma abordagem de cuidados em saúde centrado no paciente, no qual os atores do processo terapêutico seriam colocados em igual patamar e os pacientes seriam habilitados a conferir a palavra final sobre o tratamento (14). Desde então, alguns estudos têm sido levados à cabo no sentido de aprimorar esse novo modelo de cuidados.

Em primeiro lugar, é necessário se ter em mente que o paciente deve ser compreendido a partir de sua dupla condição: a de vulnerável e a de ator central no processo terapêutico (15). Relativamente a esse último aspecto, cumpre esclarecer que o cuidado centrado no paciente é um dos princípios que compõem o arcabouço teórico dos Direitos Humanos dos Pacientes, ao lado da ideia de dignidade humana, e dos princípios da autonomia relacional e da responsabilidade dos pacientes, e sinaliza no sentido de que o paciente é o agente fundamental e o principal beneficiário dos cuidados (15). O cuidado centrado no paciente envolve enfoques multiprofissionais; parcerias; respeito aos desejos do paciente a partir de suas necessidades social, emocional e física; apoio; informação; comunicação; participação do paciente e de sua família; estímulo; respeito à dignidade e à privacidade; busca de conforto físico e emocional. Assim, o paciente deve ser envolvido na terapêutica, ouvido, informado, considerado em suas necessidades físicas e emocionais pelos profissionais da saúde, que devem com o paciente estabelecer uma relação de parceria capaz de diminuir as assimetrias das relações e garantir a participação do doente nas tomadas de decisão sobre os rumos terapêuticos (15).

O cuidado centrado no paciente é uma relação valiosa que deve ser estabelecida no âmbito pessoal, profissional e organizacional e que se baseia no respeito ao paciente, considerado como ser único e individualizado, e na obrigação do profissional de velar por ele, ajudando-o a desempenhar papel mais ativo no curso do tratamento (12). Para tanto, o modelo requer empatia e profissionais da saúde mais atentos e informativos, capazes de 
abandonar o status de autoridade para estabelecer uma relação de parceria, solidariedade e colaboração (12). A confusão sobre o real sentido dos cuidados centrados no paciente pode produzir efeitos contrários aos esperados, ou seja, uma relação de cuidados não é centrada no paciente se não buscar fortalecer o relacionamento entre os envolvidos, promover a comunicação, ajudar o paciente a conhecer melhor sua saúde e a se envolver em seus próprios cuidados (12).

Apesar do entusiasmo das instituições de saúde em geral pela adoção de políticas de cuidados centrados no paciente, colocar tais políticas em prática ainda é um desafio (11). Pondera-se que cuidado centrado no paciente é uma concepção holística, baseada em relações particularizadas entre o profissional da saúde e o paciente (16), ou seja, cada paciente tem uma identidade única, relacionada com sua experiência, o meio em que vive e sua condição física e social. Desse modo, é o paciente quem deve nortear os cuidados a serem a ele oferecidos e por ele recebidos (11). Um estudo observacional verificou que os cuidados centrados no paciente envolvem comunicação, parceria, aproximação, promoção da saúde e interesse na vida do paciente (13), e que a partir da aplicação concreta de tais requisitos é possível apurar uma maior satisfação por parte dos pacientes, uma diminuição dos sintomas e menores queixas (11).

Com base nos estudos acima apresentados vê-se, portanto, que é consenso entre os pesquisadores que os cuidados centrados no paciente envolvem: a) simetria nas relações humanas e parceria entre seus componentes; b) comunicação entre os integrantes da relação e compartilhamento de informação; c) participação do paciente e de familiares nas decisões relativas ao cuidado; d) respeito aos valores e crenças do paciente. Apesar disso, existem posições diferenciadas sobre como se atingir tais padrões de cuidado, ensejando posicionamentos que valorizam aspectos diferentes do paciente e da relação em saúde.

A partir da sistematização de 34 artigos científicos que versam sobre o tema e que foram publicados entre os anos de 2004 e 2014, Pluut (13) verificou a existência de três discursos que tratam de cuidados centrados no paciente, os quais foram sintetizados da seguinte forma (12): para a primeira corrente, denominada de "Discurso 1: cuidando dos pacientes", o cuidado deve ser centrado no paciente para aliviar vulnerabilidades, amenizar sofrimentos e melhorar a qualidade dos diagnósticos. As palavras centrais para definir paciente são: necessidade de cuidados; vulnerabilidade; e experiência individual. O papel do profissional da saúde é o de cuidar e é ele o principal responsável pelas tomadas de 
decisão. A troca de informações sobre a saúde do paciente tem a função de melhorar o diagnóstico e facilitar que o paciente entre em conformidade com os conselhos médicos.

Diferentemente do primeiro discurso, para a corrente intitulada "Discurso 2: empoderando os pacientes", o objetivo é facilitar a autogestão e promover o respeito à autonomia. O paciente é encarado como alguém que tem direito ao controle dos cuidados, que é capaz de tomar decisões e exercer a autonomia de forma ampla. Os profissionais da saúde, para habilitar o paciente, devem facilitar, aconselhar e treinar o doente a decidir, bem como desenvolver a vontade e o potencial do paciente para tomar decisões. $O$ paciente, para tal vertente, é o primordial responsável pelas decisões e pode optar por compartilhar responsabilidades. Com relação às informações sobre a saúde, essas são fundamentais para um bom diagnóstico, bem como para facilitar as escolhas do paciente.

Por fim, o "Discurso 3: sendo responsivo" enfatiza a diversidade de identidade dos pacientes e de contexto das doenças, e destaca a necessidade de se centrar no processo de comunicação. Aponta que existem múltiplas identidades e variadas preferências entre os pacientes, razão pela qual o papel do profissional da saúde é se amoldar e aceitar as necessidades, valores e desejos de cada paciente no contexto do tratamento, atentandose para o estilo da comunicação e o desejo de envolvimento do doente na tomada de decisões. Tanto o paciente quanto o profissional da saúde seriam responsáveis por decidir sobre o curso do tratamento e, quanto às informações, essas seriam adaptadas a cada paciente e situação, podendo servir para obter a conformidade do paciente com o tratamento ou incentivar outras escolhas, havendo casos mesmo de possibilidade ou necessidade de retenção de informação, a depender da vontade do paciente e do respectivo contexto.

No que diz respeito à diversidade de discursos, defende-se que esta é inevitável e ao mesmo tempo valiosa, pois incita ao diálogo e à busca de melhores padrões de cuidados, dentro de uma cultura que deve estar atenta às diferentes expectativas e necessidades dos pacientes, bem como às intervenções médicas disponíveis (13). Desse modo, muito se teria a ganhar com a inexistência de um modelo único de cuidados centrados no paciente, pois, conjugando as diferentes orientações, é possível vencer o desafio de lidar com as diversidades de forma mais produtiva (13).

A conjugação de alguns elementos contidos nos três discursos apresentados por Pluut pode potencializar os efeitos benéficos dos cuidados centrados no paciente. É 
preciso que, de fato, se reconheça o paciente como um ser vulnerável, que necessita ser protegido e aliviado em seu sofrimento e cujo tratamento deve considerá-lo de forma integral. Por outro lado, não se pode crer que as decisões devam ser confiadas apenas aos profissionais da saúde e que o compartilhamento de informações sirva somente para melhorar o diagnóstico ou como subsídio para que o profissional convença o paciente a se amoldar às suas convicções sobre o tratamento. Ao invés de ocupar uma posição passiva, o paciente deve ser estimulado a gerir sua vida e exercer sua autonomia, a partir de um suporte e de um canal de comunicação adequados a habilitá-lo e a estimulá-lo a se integrar ao processo decisório.

Ao mesmo tempo, não se pode pretender que o paciente tome decisões isoladamente, sem o apoio dos profissionais da saúde e de seus familiares, e que assuma sozinho a responsabilidade de suas escolhas, pois nem sempre o paciente sabe o que quer ou o que é melhor para si, justamente por estar vivendo momentos de grande fragilidade. Para que o tratamento seja centrado no paciente de forma eficaz, deve-se ter em vista que cada ser é único e enfrenta a doença de forma individualizada. Por isso, reconhecendo as diversidades das pessoas e atentando-se para as variadas situações e preferências, as atitudes dos profissionais devem ser moldadas considerando as particularidades de cada paciente, de maneira a responder aos seus anseios, necessidades, valores e preferências no contexto de cada encontro. A comunicação deve ser adaptada ao grau de entendimento de cada paciente, a troca de informação deve servir para possibilitar escolhas, e o processo de decisão deve respeitar a vontade do paciente de se envolver. O compartilhamento e a cumplicidade que se espera num modelo eficaz de cuidados centrados no paciente não devem ser impostos.

Embora a pretensão de Pluut (13) tenha sido destacar as diferenças dos discursos, as teorias apresentadas não são excludentes, ao contrário, trazem elementos capazes de se conjugar e alicerçar um modelo de cuidado centrado no paciente que contemple a complexidade dos cuidados em saúde. Na verdade, não há preponderância de um sobre o outro, verificam-se pontos em comum entre as correntes e a convergência mais evidente é a de que o paciente deve ser considerado o ser mais importante dos cuidados e reconhecido em sua vulnerabilidade.

Portanto, defende-se nesse trabalho a adoção de um modelo de cuidado centrado no paciente que o reconheça a partir de sua vulnerabilidade e que leve em conta os seus 
aspectos individuais. Para que seja encarado como ator central, a relação de cuidados deve ser compartilhada, garantindo-se a comunicação entre os envolvidos de acordo com a capacidade de cada paciente, bem como motivando-se o doente, por meio de uma rede de proteção adequada às suas necessidades, a exercer sua autonomia. A personalização dos cuidados e a capacitação do doente para ser agente ativo da terapêutica mostram-se de especial relevância para o paciente idoso, que está sujeito a receber cuidados mais constantes e apresenta maior dificuldade em se envolver nas terapêuticas. Passa-se, então, à discussão sobre os cuidados em saúde que se entende adequado ao paciente idoso, levando-se em conta suas especificidades físicas e mentais, bem como sua especial condição de pessoa vulnerável.

\section{A aplicação do modelo do cuidado centrado no paciente à pessoa idosa}

As dificuldades na aplicação de um modelo de cuidado centrado no paciente se agravam em relação às pessoas idosas, em razão das complexidades adicionais presentes nesse grupo etário. Os pacientes idosos têm uma fragilidade acrescida pela idade, apresentam maior embaraço para compreender e participar das terapêuticas, sofrem perdas auditivas, visuais e cognitivas, estão mais propensos a ceder à pressão dos profissionais da saúde e dos familiares, são postos ao largo do processo de cuidados e são vítimas comuns de posturas paternalistas. Some-se a isso a formação do profissional em saúde, em que se prioriza o saber técnico em detrimento de aspectos pessoais, e o modelo de atendimento que requer agilidade nas consultas, incompatível com o perfil do paciente idoso, que leva mais tempo para entender e se manifestar sobre as terapêuticas. Diante de tantos fatores adversos, questiona-se se é possível envolver nos cuidados um paciente que tem a capacidade cognitiva diminuída, dificuldades de se comunicar e de se fazer entender.

De acordo com Berlinguer (6), a condição de sadio ou doente é influenciada por relações de poder e, muitas vezes, por ignorância ou preconceito, algumas condições pessoais são usadas para marginalizar o paciente. A situação do paciente idoso é emblemática, pois em razão de sua especial condição de vulnerabilidade é segregado do processo de cuidados por ser rotulado como pessoa incapaz de compreender a doença e tomar decisões sobre o tratamento. O fato é que a doença "é revestida de um juízo social 
pelas consequências que provoca na vida cotidiana" (6), daí porque determinados comportamentos podem agravar, ao invés de minorar, os efeitos da doença (6).

Reconhece-se que no cuidado centrado no paciente busca-se uma parceria personalizada entre os envolvidos, sendo importante que, além do compartilhamento dos cuidados com o profissional da saúde e familiares, leve-se em conta o que é realmente significativo para cada paciente (11). Com relação aos pacientes idosos, a consecução de um tratamento compartilhado se apresenta mais trabalhoso porque o profissional da saúde nem sempre compreende os seus desejos e expectativas. Normalmente, a vontade do paciente idoso é colocada em segundo plano e ele é vítima comum de obstinações terapêuticas indesejadas, principalmente em fase de terminalidade da vida.

Para que se possa pensar num modelo de cuidado que erija a pessoa idosa à alçada de protagonista do seu tratamento, é preciso, primeiramente, que ela seja reconhecida como pessoa vulnerável, mas não de uma forma depreciativa, capaz de gerar posturas paternalistas, ao contrário, de maneira a se criar mecanismos de proteção e de compensação das perdas físicas e cognitivas para que possa desenvolver suas capacidades. O paciente idoso não deve ser encarado apenas como um ser vulnerável que precisa de cuidados (9). Ainda que dependente de terceiros, o paciente idoso também busca "experiências e sensações que os fazem sentir-se vivos, ao invés de serem considerados pessoas de segunda classe cuja existência se reduz às suas condições biológicas" (8). Assim, o cuidado centrado no paciente idoso deve conjugar o reconhecimento de sua vulnerabilidade à necessidade de se capacitá-lo para que possa, de forma autônoma, participar da tomada de decisões sobre sua terapêutica.

O simples fato da idade não inabilita o paciente idoso a fazer escolhas pessoais que traduzam seus próprios desejos, valores e concepções de vida. Por tal motivo, os profissionais de saúde, cuidadores e familiares não podem menosprezar a vontade da pessoa idosa ou desconsiderar as ocasiões em que ela precisa do respaldo de seus relacionamentos para se manifestar ou tomar decisões (15).

Uma ocasião em que se observa dificuldades na aplicação de cuidados centrados no paciente refere-se às situações de terminalidade da vida, em que os profissionais de saúde devem ter, além de habilidades e conhecimentos, capacidade de responder às necessidades espirituais e emocionais dos pacientes (18). Para isso, é necessário discutir o processo de envelhecimento e de adoecimento entre os envolvidos, esclarecer e 
informar o paciente para que, consciente e fortalecido, possa ter respeitadas sua autonomia e dignidade no fim da vida (14). Pelluchon (9) aborda a extrema vulnerabilidade de pacientes idosos no final da vida e destaca que, a despeito disso, o profissional da saúde deve ouvi-lo, ser sensível às suas especiais necessidades, providenciar informações de uma maneira que o paciente possa entendê-las, ser honesto e inspirar confiança. A opção por determinada conduta em saúde em fase de terminalidade não pode ser fruto de um processo de discriminação que leva uma pessoa vulnerável a sentir que não merece viver quando se torna idosa. Ao contrário, cada ser humano, mesmo quando não detém mais as funcionalidades físicas ou capacidade cognitiva plena, possui um valor intrínseco, razão pela qual deve ser respeitado e tratado com dignidade. É dever do profissional da saúde ajudar o doente a restaurar sua autoestima, reconhecer-se como ser humano e como ator central do processo de cuidados. A atenção à vulnerabilidade da pessoa idosa em final de vida é elemento central nos cuidados paliativos, por exemplo (9). Assim, para centrar os cuidados no paciente idoso, os profissionais da saúde e familiares devem ajudálo não apenas a sobreviver, mas a existir a despeito de suas limitações, isto é, não se pode reduzir o paciente idoso às suas incapacidades, ao contrário, devem-se buscar maneiras pelas quais ele possa transcendê-las (9).

\section{Considerações finais}

O adoecer é uma experiência individual e única, que fragiliza e compromete a vida das pessoas, por isso deve ser enfrentado de forma personalizada e combatido mediante tratamentos que tenham em vista não só a doença, mas o paciente considerado em seu todo. Longe de ser a doença a protagonista das terapêuticas, é o doente quem deve exercer esse papel, ou seja, deve ser o ator central dos cuidados, a quem devem ser dirigidas atenções que levem em conta seus anseios, medos, formas de encarar e enfrentar a doença, grau de educação, nível cultural e meio social em que vive.

Embora haja reconhecimento da importância de se centrar os cuidados no paciente, encarando-o como o ator principal do tratamento e a ele dispensando os melhores cuidados em saúde, a estipulação de um modelo amplamente aceito de centralidade dos cuidados mostra-se uma tarefa complexa, em razão das peculiaridades de cada paciente, das diferentes necessidades e desejos, do modo diverso de se encarar a doença, bem como em função do modelo tradicional de cuidado que ainda se encontra arraigado em 
nossa cultura. Apesar disso, reconhece-se a necessidade de mudança de posturas paternalistas e assimétricas, e há significativas evidências de que centrar os cuidados no paciente tem efeitos positivos em sua melhora.

A despeito da discriminação do paciente idoso, é possível centrar os cuidados na pessoa idosa que tem a capacidade cognitiva diminuída e dificuldades de se comunicar. A senilidade não justifica a supressão da vontade, o abandono das tentativas de envolver o paciente idoso, tampouco chancela posturas paternalistas. Assim, rechaça-se a noção de uma medicina baseada em relações de poder dos profissionais da saúde, bem como se repudia a ideia de que o envelhecer possa se transformar num julgamento social. $O$ paciente idoso muitas vezes é visto como um paciente incômodo e seus problemas de saúde se agravam por insensibilidade humana. É necessário que haja uma mudança de mentalidade no sentido de promover a integração, e não a discriminação, mediante ampliação dos conhecimentos científicos acerca das condições peculiares de saúde da pessoa idosa, conscientização social sobre a importância dos cuidados centrados no paciente idoso, além de um amadurecimento da legislação e a instituição de políticas públicas voltadas à inserção do paciente idoso no tratamento.

\section{Referências}

1. Bensing J. Bridging the gap: the separate worlds of evidence-based medicine and patient-centered medicine. Patient Education and Counseling, 2000, 39(1):17-25.

2. Hughes JC, Bamford C, May C. Types of centredeness in health care: themes and concepts. Medicine, Health Care and Philosophy, 2008, 11(4):455-463.

3. Stempsey, WE. Hope for health and health care. Medicine, Health Care and Philosophy, 2015, 18(1):41-49.

4. Gustavsson E, Sandman L. Health-care needs and shared decision-making in prioritysetting. Medicine, Health Care and Philosophy, 2015, 18(1):13-22.

5. World Health Organization. Patient's rights. Disponível em: http://www.who.int/genomics/public/patientrights/en/. Acesso em 8.6.2017.

6. Berlinguer G. A Doença. São Paulo: Hucitec/CEBES,1988.

7. Berlinguer G. Ética da saúde. São Paulo: Hucitec/CEBES; 1996. 
8. Mishler EG. The discourse of medicine: dialectics of medical interviews. New York: Ablex Publishing Corporation, 1984.

9. Pelluchon C. Taking vulnerability seriously: what does it change for bioethics and politics? In: Masferrer A; García-Sánchez, E, editors. Human dignity of the vulnerable in the age of rights. Valencia: Springer, 2016.

10. Shaller, D. Patient-centered care: what does it take? The Commonwealth Fund, 2007. Disponível em: https://psnet.ahrq.gov/resources/resource/6254/patient-centered-carewhat-does-it-take Acesso em 9.2.2017].

11. Ells C, Hunt MR, Evans JC. Relational autonomy as an essential component of patientcentered care. International Journal of Feminist Approaches to Bioethics, 2011, 4(2):79101.

12. Epstein RM, Street RL Jr. The Values and Value of Patient-Centered Care. Annals of Family Medicine, 2011, 9(2):100-103.

13. Pluut B. Differences that matter: developing critical insights into discourses of patientcenteredness. Medicine, Health Care and Philosophy, 2016, 19(4):501-515.

14. Joterrand F, Amodio A, Elger B.S. Patient education as empowerment and selfrebiasing. Medicine, Health Care and Philosophy, 2016, 19(4):553-561.

15. Albuquerque A. Direitos Humanos dos Pacientes. Curitiba: Juruá, 2016.

16. Stewart M. Towards a global definition of patient centered care. BMJ, 2001, 322(724): 444-45.

17. Little P, Everitt H, Williamson I, Warner G, Moore M, Gould C, et al. Observational study of effect of patient centeredness and positive approach on outcomes of general practice consultations. BMJ, 2001, 323(7318):908-11.

18. Poletto S, Bettinelli LA, Santin JR. Vivências da morte de pacientes idosos na prática médica e dignidade humana. Revista Bioética, 2016, 24 (3): 590-595.

\section{Como citar este artigo:}

Paranhos DGAM, Oliveira AAS. O modelo de cuidado centrado no paciente sob a perspectiva do paciente idoso. Revista Cadernos Ibero-Americanos de Direito Sanitário. 2018 abr./jun, 7(2):95-109. 